\title{
REVISTA DE INVESTIGACIÓN EN SALUD. UNIVERSIDAD DE BOYACÁ
}

2018;5(2):323-344. doi: https://doi.org/10.24267/23897325.346

\section{ARTÍCULO DE REVISIÓN}

\section{El acompañamiento por enfermería en la atención del trabajo de parto y parto: una revisión narrativa de la literatura}

\author{
Nursing accompaniment in labor and delivery care: A narrative \\ review of the literature \\ A Assistência de enfermagem no cuidado de parto e parto: uma \\ revisão narrativa da literatura.
}

\begin{abstract}
Ariadna María Angarita Navarro ${ }^{1 *}$
${ }^{1}$ Fundación Universitaria del Área Andina. Seccional Bogotá, Colombia

*Correspondencia: Dirección: Cra. 14A \#70A-34, Bogotá Celular: 3102188303

Correo electrónico: ariadna.angarita@gmail.com.
\end{abstract}

\section{Citar este artículo así:}

Angarita-Navarro AM. El acompañamiento por enfermería en la atención del trabajo de parto y parto: una revisión narrativa de la literatura. Revista Investig Salud Univ Boyacá. 2018;5(2) 


\section{RESUMEN}

Introducción. El trabajo de parto y parto es un acontecimiento importante en la vida de las mujeres y su familia; es así, como entre angustia, miedo, ansiedad y contracciones, la mujer espera ser cuidada, sentirse protegida y acompañada. Por esta razón, es fundamental resaltar el rol de enfermería en este suceso relevante, siendo necesario actuar desde la humanización, proporcionándole a las madres los cuidados correspondientes para que se sientan a gusto y satisfechas.

Objetivo. Realizar una revisión de la literatura disponible acerca de los beneficios del acompañamiento por parte del profesional de enfermería en el proceso de parto y parto.

Método. Revisión documental mediante la búsqueda de la literatura de los últimos 10 años, disponible en las bases de datos Science direct, EBSCO, Ovid en el período de marzo a agosto de 2017. Como criterios de inclusión se tuvo en cuenta: acompañamiento del profesional de enfermería en el trabajo de parto y parto, artículos de investigación publicados en los últimos 10 años, y criterios de exclusión (acompañamiento en el trabajo de parto y parto por la pareja, familia, doulas y matronas).

Resultados. Algunos de los artículos revisados y analizados indican que hace falta acompañamiento por parte del profesional de enfermería a las mujeres en trabajo de parto y parto; otro grupo de artículos determinan que es fundamental el cuidado de enfermería en este proceso de trabajo de parto y parto, debido a que son los profesionales que orientan e informan a las parturientas y familiares sobre la situación de la paciente y el hijo por nacer, además de enseñar cuidados que fortalezcan el rol materno.

Conclusión. Existen diferentes factores que han contribuido a la deshumanización del cuidado de enfermería, por tanto, es necesario fortalecer desde la academia la formación del futuro profesional de enfermería, de tal forma que tenga en cuenta tanto el conocimiento científico como la competencia del saber ser.

Palabras clave: parto, trabajo de parto, parto humanizado, atención de enfermería, cuidado de enfermería. 


\begin{abstract}
Introduction. Labor and delivery are a significant event for both women and families; therefore, amidst women's anguish, fear and contractions, they expect to feel protected and accompanied. For this reason, it is essential to highlight the nurses' role in this relevant occasion, nursing professionals must act from a humanizing perspective by providing mothers the corresponding care so they feel comfortable and satisfied.
\end{abstract}

Objective. To conduct a review of the available literature about the benefits of the nursing professional accompaniment during labor and delivery.

Method. A documentary review through the search of literature from the past 10 years, available on the databases: Science direct, EBSCO, Ovid during the period corresponding to march and august 2017. The following inclusion criteria were taken into account: accompaniment of the nursing professional during labor and delivery care, articles published within the last 10 years, and the exclusion criteria were: accompaniment of the spouse, family, doulas and midwives.

Results. One set of the articles reviewed and analyzed indicate the lack of accompaniment by the nursing professional to women in labor and delivery; a different set of articles determine that the nursing care is fundamental during the labor and delivery process since the professionals are in charge of guiding and informing both women in labor and relatives about the patient and the yet-to-be-born child's situation, their role also involves teaching mothers about the necessary care in order to strengthen their maternal role.

Conclusion. There are different factors which may have contributed to the dehumanization of nursing care; therefore, it is necessary to strengthen from the academy the training of future nursing professionals, in such a way that they possess both the scientific knowledge and the competences related to human development.

Key words: Delivery, Labor, Humanizing Delivery, Nursing Care 


\section{RESUMO}

Introdução. O trabalho de parto e parto é um evento importante na vida das mulheres e suas famílias; é assim que, entre angústia, medo, ansiedade e contrações, a mulher espera ser cuidada, sentir-se protegida e acompanhada. Por esse motivo, é fundamental destacar o papel do enfermeiro nesse evento relevante, sendo necessário atuar a partir da humanização, proporcionando o cuidado correspondente para que as mães se sintam confortáveis e satisfeitas.

Objetivo. Realizar uma revisão bibliográfica sobre os benefícios do acompanhamento pelo profissional de enfermagem no processo de parto e parto.

Método. Revisão documental, pesquisando no período de março a agosto de 2017 na literatura disponível nas bases de dados Science direct, EBSCO e, Ovid,. Os critérios de inclusão foram: acompanhamento do profissional de enfermagem no trabalho de parto e parto, artigos de pesquisa publicados nos últimos 10 anos; e critérios de exclusão: acompanhamento no trabalho de parto e parto pelo casal, família, doulas e parteiras.

Resultados. Alguns dos artigos revisados e analisados indicam que há falta de apoio do profissional de enfermagem às mulheres no trabalho de parto e parto; outro grupo de artigos determina que o cuidado de enfermagem é fundamental no processo de parto e parto, pois é esse o grupo de profissionais que orienta e informa às parturientes e familiares sobre a situação do paciente e do filho, além de ensinar o cuidado que fortalece o papel materno.

Conclusão. Existem diferentes fatores que têm contribuído para a desumanização da assistência de enfermagem; assim, é necessário fortalecer as Faculdades para que a formação do futuro profissional de enfermagem não leve em consideração apenas o conhecimento científico, mas também a competência do saber ser.

Palavras-chave: parto, trabalho de parto, parto humanizado, cuidados de enfermagem. 


\section{INTRODUCCIÓN}

Existen muchas profesiones que implican el cuidado, considerándose esta acción como inherente al ser humano. La real academia española define cuidar como "asistir, conservar, cautela, amenaza, intranquilidad, advertencia en proximidad de peligro o la contingencia de caer en error, vivir con advertencia respecto de una cosa, atención, solicitud, guardar, preocuparse, prestar atención a algo o a alguien" (1); sin embargo, el cuidado es la esencia de la profesión y disciplina de enfermería (2), es así, como la ley 266 de 1996 que reglamenta la profesión en Colombia, manifiesta que la enfermería "tiene como fin dar cuidado integral de salud a la persona, a la familia, la comunidad y a su entorno" (3). Por otro lado, dentro del metaparadigma de la profesión, cada teoría de enfermería alimenta desde su perspectiva el concepto de cuidado; así, en un artículo escrito por Daza Caballero, Rosita y Medina, Luz Estela, se afirma que "el cuidado no tiene un concepto único, ni homogéneo, se encuentra una tendencia hacia una acción encaminada hacia la recuperación del estado de bienestar" (4). De acuerdo con esto, se puede determinar que el cuidado de enfermería es definido dentro del metaparadigma de cada teoría de enfermería, con el objetivo final que los profesionales de enfermería se comprometan con el cuidado, reflejándose en la satisfacción de los pacientes con respecto a la atención, el uso de la tecnología, la capacidad para prevenir complicaciones en ellos, el apoyo y la actitud (5).

La enfermería cuenta con un sin número de conocimientos científicos, que posiblemente han sido subvalorados por décadas, posiblemente por no saber cómo aplicarlos en la práctica, o tal vez, el interés ha sido siempre el modelo biomédico. A lo largo del tiempo, se ha observado que la enfermería ha estado enfocada en el cumplimiento de otras funciones enlazadas con el actuar médico, desvirtuando su quehacer; ante esta situación Madeleine Leininger, en los años 50 , se pronunció refiriendo: "Alenté a las enfermeras para que mantuvieran las primeras conferencias sobre la ciencia de enfermería, siendo la palabra teoría un nuevo reto para muchas enfermeras, fue vista como -cosas de la torre de marfil-. Muchas enfermeras y médicos no pudieron ver la utilidad de las teorías de enfermería, pues como enfermeras eran -hacedoras y prácticas- y se esperaba principalmente que cumplieran las órdenes de los médicos, manteniendo la antigua cultura de la enfermería" (6). Aunque han pasado varias décadas desde la afirmación de Leininger en los años 50's, aún es posible identificar dicho actuar en los profesionales de enfermería. De igual forma Durán de Villalobos realiza una crítica al cuidado que se ha venido llevando a cabo: "Se dice que cuidar es la esencia de la enfermería, y que el cuidado es el elemento paradigmático que hace la diferencia entre enfermería y otras disciplinas del área de 
la salud. Pero no se puede pasar por alto el que muchas enfermeras enfatizan más en los aspectos relacionados con la curación, y han mantenido sus intervenciones más hacia la línea del curar que en la del cuidar" (7).

Por otro lado, desde el área materno perinatal, a diferencia de otros países, en Colombia, el grupo de profesionales de enfermería que cuida de las gestantes en los controles prenatales no es el mismo que cuida de ellas en los servicios de ginecobstetricia; mientras que en los controles prenatales y en los cursos de preparación para la maternidad y paternidad responsable se educa y se prepara a la gestante y a su familia para una experiencia positiva, las expectativas cambian al ingresar a los servicios de salud para la atención del parto, pues el acompañamiento de los profesionales en enfermería puede que no sea el adecuado ni el que se esperaba; es así, como en un sin número de situaciones, son las gestantes y sus familiares quienes piden dicho acompañamiento, solicitud que no debería ser necesaria ya que se trata de un actuar es inherente al cuidado de enfermería (8).

Aunque de ninguna manera son justificables algunas acciones relacionadas con la deshumanización en la atención y el cuidado por parte de los profesionales en enfermería, es claro que existen varios factores que impiden el acercamiento, la empatía y el acompañamiento del profesional de enfermería a sus pacientes; actualmente concurre una problemática relacionada con la asignación del rol administrativo a los profesionales de enfermería, rol que incita a delegar el cuidado a los auxiliares para que el profesional se ocupe con tareas asignadas desde un escritorio. Es así, como el cuidado integral se debe convertir en el norte de todo enfermero o enfermera en salas de parto, de tal forma que en su actuar puedan tener la capacidad de identificar las necesidades de las gestantes y sus familias, y así, cuidar respetando el género, la cultura, el contexto social y, mediante la empatía, convertirse en una persona significativa para la paciente y la familia que anhelan la llegada de un nuevo integrante (9).

Se ha reconocido que durante el trabajo de parto y parto se presentan el miedo y la ansiedad; no solo la madre tiene estas emociones, la familia y allegados también los padecen; sienten el miedo de lo que pueda pasar en el parto, miedo ante complicaciones de la madre y el recién nacido, miedo a malformaciones, entre otras, aumentando igualmente la ansiedad (10). De esta forma, durante la gestación, trabajo de parto, y parto, la gestante y la familia se encuentran a la expectativa, sus pensamientos y muchas de sus decisiones giran en torno al hijo por nacer; el sentimiento de ansiedad se convierte en el protagonista debido a la incertidumbre que les genera pensar en un futuro con el nuevo integrante de la familia (11); pero es desde la enfermería que se puede ayudar a mitigar estas emociones, con sus cuidados, con 
el acompañamiento que incluye brindar información adecuada, contribuyendo a vivir este suceso de una forma positiva (12).

En el pasado, el parto se vivía de una forma diferente, era atendido en la casa en compañía de la familia; es relevante destacar que esta práctica tenía como aspecto positivo el acompañamiento y el apoyo por familiares y allegados a la mujer, brindándole calor humano y sensación de bienestar (11). Con el tiempo, a partir de los años 60 aparecen grandes hospitales, llevando a que los partos empezaran a ser atendidos en dichas instituciones hospitalarias por profesionales de salud, de esta manera se logró disminución de muertes materno-perinatales, sin embargo, el acompañamiento que tenían las mujeres cuando el parto era atendido en casa se ha perdido (11).

Por otro lado, al enfocar el acompañamiento de la enfermería durante el trabajo de parto y parto, es necesario discutir un poco sobre la violencia obstétrica desde el rol que lleva a cabo el enfermero(a) profesional; estudios al respecto referencian que "en cuanto a las personas que las usuarias identifican como principales perpetradores del trato deshumanizado, tenemos al personal de enfermería, hasta en un $70 \%$ de los casos" (13). En Colombia se ha venido trabajando en la eliminación de la violencia obstétrica (14), por lo tanto, el rol que debe tomar el profesional de enfermería es abogar y apoyar a la paciente; es claro que estos actos de violencia obstétrica en Colombia están directamente relacionados con violencia de género (15), y se presentan cuando "se le niega a la gestante la posición que desee en el parto, cuando obstaculizan el apego del recién nacido con la madre, en la administración de oxitocina para acelerar el parto, cuando se realizan enemas o rasuración de región pélvica, al tener un acceso con líquidos endovenosos sin necesidad, impidiendo la libre deambulación, al prohibir la compañía de la parturienta en el trabajo de parto y parto, y negarse a brindar información a las pacientes y familiares"(16).

En la búsqueda previa a la revisión bibliográfica, sobre la definición "acompañamiento", se encontró el artículo titulado "Acompañamiento y cuidado holístico de enfermería en personas con enfermedades crónicas no adherentes al tratamiento" en el cual se determina que "la palabra acompañamiento se relaciona con pensar en una persona a quien se acompaña como ser pasivo y que no puede valerse por sí... no obstante, el concepto abarca más allá de la entrega de indicaciones: significa que el profesional de enfermería entienda que la persona acompañada es la protagonista y que el acompañante debe adaptarse a sus necesidades, demandas, prioridades y valores"(17).

Es así que el cuidado que abarca el acompañamiento de la enfermería, está enfocado en ofrecerle bienestar a la mujer, suplir las necesidades 
que caracterizan la situación o la vivencia que ellas tienen, brindarles apoyo, escucharlas y estar presentes o en contacto con cada una de ellas (18).

El acompañamiento desde la enfermería en trabajo de parto y parto implica contribuir para que la mujer se sienta la protagonista de una experiencia especial y emocionalmente positiva (19).

Entonces, teniendo en cuenta el concepto de acompañamiento de la enfermería, es transcendental el cuestionamiento sobre el quehacer de enfermería y sus repercusiones en el presente. Por ello, como sujetos morales, se deben generar y aprovechar espacios de deliberación con el propósito de asumir verdaderos compromisos para el cambio, la toma de conciencia moral y el fortalecimiento de la profesión (20).

Por esta razón, el objetivo del estudio consistió en realizar una revisión de la literatura disponible acerca del acompañamiento por parte del profesional de enfermería en el proceso de parto y parto, durante el período de marzo a agosto del año 2017.

La metodología fue realizar una revisión bibliográfica (21) del acompañamiento desde la enfermería en el trabajo de parto y parto. Se hizo una búsqueda en bases de datos Science direct, EBSCO y Ovid; filtrando los últimos diez años, la cual se llevó a cabo desde marzo hasta agosto del año 2017, utilizando descriptores o DeCS: parto, trabajo de parto, parto humanizado, atención de enfermería.

Se tuvieron en cuenta criterios de inclusión como acompañamiento del profesional de enfermería en el trabajo de parto y parto, asimismo, se usaron los DeCS en la búsqueda de artículos de investigación y artículos publicados en los últimos 10 años; los criterios de exclusión fueron: acompañamiento en el trabajo de parto y parto por la pareja, familia, doulas y matronas. Se trabajó bajo una matriz documental realizada en Excel, en la cual se analizaron artículos de investigación según la pertinencia y el cumplimiento con los criterios de inclusión y exclusión; obteniendo una muestra final de 60 artículos los cuales fueron organizados en la matriz bibliográfica, donde se clasificaron los artículos seleccionados por título, base de datos, autor, tipo de documento, resumen del artículo, palabras clave, fecha de publicación, dirección URL, tipo de estudio, resultados, bibliografía consultada por el autor, descripción del documento y cita en norma Vancouver o APA.

En la búsqueda bibliográfica se encontró una variedad de artículos relacionados con el acompañamiento por parte de las doulas, las matronas y familiares de la mujer gestante, pero pocos de acompañamiento por parte del profesional de enfermería; además, la palabra acompañamiento no es un término DeCS, y al buscar cuidado de enfermería con relación al trabajo de parto y parto, 
los resultados de la búsqueda tienen otra dirección, pues estos tienden a ser muy generales y no muchos de estos artículos mencionan el acompañamiento de enfermería en dicha circunstancia.

\section{ASPECTOS ÉTICOS}

El presente estudio de revisión hace parte de un macroproyecto Ilamado Cuidado de enfermería en el proceso de gestación, referenciado en la literatura científica de los últimos 10 años; proyecto aprobado por el Comité de Investigación de la Fundación Universitaria del Área Andina como proyecto institucional.

Esta investigación, según la resolución 8430 de 1993 expedida por el Ministerio de Salud, se considera investigación sin riesgo, al ser un estudio que emplea un método de investigación documental retrospectiva y no se realiza ninguna intervención o modificación intencionada de las variables biológicas, fisiológicas, psicológicas o sociales de los individuos (22).

\section{RESULTADOS}

Tras la revisión bibliográfica en diferentes bases de datos, se evidenció que las gestantes, durante su trabajo de parto, requieren el acompañamiento del profesional de enfermería
Una investigación realizada por Lagos Jiménez (2016), indica que las condiciones laborales de los profesionales de enfermería no han sido adecuadas para acompañar individualmente a cada mujer que llega a trabajo de parto, además de las barreras como cambios de turnos, que muchas veces no permiten continuidad en el cuidado; además, refiere que aunque el profesional en enfermería pudiese dedicarle tiempo a una sola mujer, necesitaría la ayuda de otro acompañante para proporcionarle apoyo físico y emocional constantes. Esta investigación motiva a reconocer la importancia de la educación que el enfermero(a) debe ofrecerle a otro acompañante, el cual puede ser escogido por la mujer, y así, en trabajo conjunto, logren proporcionarle bienestar a la embarazada durante el trabajo de parto y parto. Este mismo autor indica que "Las mujeres deberían contar con personas próximas y profesionales que les apoyen durante el trabajo de parto y nacimiento; mejora la fisiología del parto y la sensación de confianza materna" (23).

Según un estudio de investigación realizado por Fróes de Oliveira Sanfelice, de Souza Freitas Abbud, Separavich Pregnolatto, Gonçalves da Silva y Keiko Kakuda Shimo, "en el trabajo de parto y parto, el profesional de enfermería juega un papel primordial puesto que será el encargado de tomar la responsabilidad y empoderamiento de la atención y el cuidado integral de la madre y el recién nacido" (24), indicando además que 
es el enfermero(a) quien toma decisiones sobre el cuidado de las gestantes; pero en la realidad colombiana se puede evidenciar que el rol del profesional en enfermería ha cambiado, pues debe cumplir funciones administrativas y seguir normas y protocolos institucionales, dejando de lado el cuidado de los pacientes, en este caso, el cuidado de las gestantes (25).

Aunque en el estudio se indica que la enfermería tiene un rol importante en la toma de decisiones en el cuidado de las pacientes, también, se hace un contraste al referir que la enfermería está relacionada con la violencia obstétrica cometida a diario contra las mujeres, por medio de palabras (24). Se considera violencia obstétrica cuando se llevan a cabo expresiones de ironía, procedimientos invasivos (uso de oxitocina sintética, episiotomía rutinaria, rasurado, realización de cesáreas innecesarias), razón por la cual las mujeres se sienten inconformes con el cuidado y la compañía brindado por enfermería (26). Es así, como enfermería debería aprovechar el rol significativo dentro de la sociedad a la hora de brindar cuidado para abogar por las pacientes en salas de parto, apoyarlas, y ofrecerles el acompañamiento que necesiten.

Por otro lado, una investigación realizada por Laura Mablmeister en el año 2007 expone que en el trabajo de parto y parto, la madre y la familia atraviesan por un estado de preocupación al no saber cómo se encuentra su hijo por nacer y las posibles complicaciones que puedan tener tanto la madre como el hijo (27); es esta la razón por la cual el profesional de enfermería debe tener una comunicación asertiva con la gestante, su pareja y su familia, lo cual disminuye la ansiedad y la preocupación y a la vez, genera un cuidado que conduce a la satisfacción de la mujer y toda su familia (28). Esto se ratifica en un estudio de tipo cualitativo, denominado "Experiencias de las mujeres durante el trabajo de parto y parto"; en dicho estudio surgieron algunas categorías, una de ellas la soledad, la cual recoge el sentir de las pacientes, quienes consideraron importante que el personal de salud les informara sobre su evolución, tanto a ellas como a sus familiares, actividad que fue tomada como una forma de acompañamiento. Percibieron el acompañamiento existente cuando el personal de salud se mostraba atento a su evolución y actuaba de inmediato a su llamado, lo cual las hizo sentir que no estaban $\tan$ solas (29).

En el artículo realizado por Cynthia Ramírez Hernández, titulado "Modelo de atención obstétrica por enfermeras obstetras y perinatales en el hospital general de Cuautitlán: una experiencia exitosa", se encontró un modelo de atención al parto por parte de los profesionales de enfermería en donde se incluían competencias como atención al parto, valoración obstétrica, inducción y conducción de trabajo parto, atención inmediata 
al recién nacido, entre otras; dando así un papel fundamental a los profesionales de enfermería en el ámbito materno-perinatal (30).

Carlos Sergio Correa dos Reis, Danielle de Oliveira Miranda de Souza y María de Fátima Hasek Nogueira realizaron una investigación llamada "Análisis de partos atendidos por enfermeras obstétricas bajo la perspectiva de la humanización del parto y nacimiento en Brasil", en ella se resalta el acompañamiento de la enfermería en el trabajo de parto, indicando que los partos acompañados por enfermería no tuvieron complicaciones, evolucionando sin acudir a la oxitocina, amniotomía, compresión del fondo uterino, reducción del cuello uterino y/o la episiotomía(31). El acompañamiento también permitió la realización de ejercicios de respiración, estímulo de la libre deambulación, uso de agua tibia para baño reduciendo el dolor, realización de masajes y el estímulo-enseñanza a un familiar o pareja para que continuara con el masaje y por ende, reducir el dolor en la mujer; además, emplearon técnicas como aromaterapia y la crioterapia (31).

De igual forma, una investigación realizada por Oria Vicharra (2007), demuestra que el acompañamiento durante el trabajo de parto ofrece ventajas para la madre, el hijo por nacer y todo el personal de salud que incluye al enfermero(a); de esta forma los partos no serían prolongados, todo lo contrario, se facilitarían disminuyendo el tiempo que transcurre en cada una de sus fases; además, disminuye la incidencia de cesáreas y el riesgo de complicaciones (32).

Las autoras Julyenne Dayse Gomes de Oliveira, Taynah Neri Correia Campo, Francisca Marta de Lima Costa Souza, Rejane Marie Barbosa Davim, Janmilli da Costa Dantas en el año 2016 dieron a conocer que el apoyo que ofrecen los profesionales de enfermería durante el trabajo de parto a las mujeres es muy importante, este se evidencia al estar cerca de ellas, por la preocupación que tienen las enfermeras(os) con respecto a la gestante en trabajo de parto, por su disposición para cuidarlas; de igual forma, la escucha ofrecida a las mujeres (33), permitió crear empatía, vínculos de confianza y afecto, generando confort para la mujer durante el trabajo de parto y parto (34).

Según Morláns en un estudio llamado "El acompañamiento continuo en los partos instrumentales: resultados obstétricos y perinatales, nivel de satisfacción de la mujer y acompañante", el papel de los profesionales de la salud es "permitir y alentar a todas las mujeres, si lo desean, a que cuenten con personas de apoyo durante todo el proceso de modo ininterrumpido, facilitando que el acompañamiento se inicie desde las etapas más tempranas del mismo, así como a promover una política institucional que le permita a la gestante elegir libremente la persona que la acompañe de forma continuada durante todo el proceso del parto" (35). Se reconoce de esta forma que el acompañamiento a la gestante por parte de 
un familiar, pareja o amigo(a) es necesario, pero son los profesionales de la salud, en este caso el de enfermería, quienes deben permitir y promocionar dicho acompañamiento a las pacientes en salas de parto.

Otro estudio titulado "Importancia del acompañamiento continuo en cesáreas programadas: ansiedad materna" refiere que en algunos casos se presentan situaciones en la gestante que la llevan a elegir el parto por cesárea, pero cuando esto pasa, las condiciones son diferentes, la sala de cirugía es fría, y existe menos contacto con el personal de enfermería. En el mismo estudio se indica que es fundamental para la mujer el acompañamiento por parte del profesional de enfermería en esta experiencia, de igual forma, permitir la presencia de una persona significativa para la gestante en dicha sala de cirugía, pues esto contribuye a disminuir la ansiedad (36).

Un estudio realizado por Octavio Muniz da Costa Vargens, Alexandra Celento Vasconcellos da Silva, Jane Márcia Progianti en el año 2016, demuestra que las enfermeras obstétricas están luchando por brindar una atención humanizada en el parto; además, en ese estudio se evidencian las diferentes prácticas empleadas por las enfermeras(os) obstétricas, entre ellas se encuentran la atención del parto y el acompañamiento en el mismo; durante el acompañamiento las enfermeras llevan a cabo cuidados para disminuir el dolor de la mujer, facilitando la respiración y disminuyendo la angustia que puede tener la mujer al desconocer su estado y el de su hijo por nacer; de igual forma, con estos cuidados se disminuye la sensación de soledad gracias a su compañía y apoyo en el trabajo de parto (37).

Contreras y Guaymás, en un artículo publicado en (2017), sostienen que "el cuidado de enfermería que hace énfasis en la humanización, tiene que ir más allá de realizar un simple procedimiento, debe permitirnos reflexionar sobre las necesidades de las mujeres que se encuentran en trabajo de parto y parto, las cuales deben ser atendidas con especial consideración por su estado de vulnerabilidad"; al respecto, también afirman que "brindar apoyo continuo a la madre durante el parto trae resultados positivos en la reducción del consumo de drogas para el dolor, parto instrumentado, duración del trabajo de parto y disminución de la tasa de cesáreas. Según lo anterior, el autor resalta la importancia del rol de enfermería, pero además determina la necesidad de asumir la profesión con responsabilidad ética, legal y moral, y no solamente técnica y científica por parte de los profesionales de enfermería" (38).

Por otro lado, algunos estudios dejan ver el rol de la enfermería desde una perspectiva negativa; es así como en un estudio llamado "Home parturition: power to feminine nature and a challenge for the obstetric nurse", se plantea que existe un 
alto porcentaje de mujeres que reportan insatisfacción en la atención del parto en relación con el cuidado del profesional de enfermería; algunas percepciones de las mujeres participantes en la investigación, están relacionadas con las expectativas que tienen respecto al cuidado por parte del personal de enfermería, indican un trato no digno y falta de solidaridad con respecto a su estado físico y emocional (39).

En relación con el trato no digno, en algunas ocasiones se puede evidenciar que el personal juzga a las pacientes en las salas de parto por distintas razones; al respecto, Cristina López Villar y Aida Soraluce Acebo afirman que en el proceso de "acompañamiento se debe comprender, aceptar, respetar, reforzar y no juzgar. La mujer durante el proceso es capaz de sentirse mucho más segura de sí misma y de sus propias capacidades instintivas para parir felizmente si tanto los profesionales como las personas que la rodean escuchan, apoyan y sostienen" (40).

Evidentemente, el cuidado de enfermería implica que el profesional recurra a los conocimientos científicos, a un trato amable y a las actitudes de atención que favorezcan el crecimiento en el cuidado a través de la interacción y la comunicación con los otros, lo cual arroja diversos beneficios en el estado físico, emocional y psicológico de la madre, y ayuda en la relación del vínculo madre-hijo (41). Por esta razón, el profesional de enfermería debe estar siempre atento al mantenimiento del respeto y la privacidad de las personas que reciben el cuidado, no solo por parte de él, sino de todo el personal que entra en contacto con una mujer durante el trabajo de parto y parto (42).

De igual forma, en la búsqueda de artículos de investigación se encontró un estudio llamado "Humanización del cuidado de enfermería durante el parto". En esta investigación se evidencia el poco reconocimiento que las gestantes tenían del personal de enfermería, pues el $48 \%$ de las participantes no reconocieron a las enfermeras durante su estadía en el hospital; según las participantes, no tuvieron la posibilidad de saber si recibieron o no cuidados por parte de dichos profesionales; probablemente el personal de enfermería no se presentó al iniciar el turno ni durante el mismo. Este estudio arroja que un $63 \%$ de las mujeres participantes manifestaron insatisfacción, pues la enfermera no había tenido ningún tipo de contacto con sus familiares para informarles como estaban ella y su hijo por nacer (43).

En otro estudio, el cual consistió en una revisión bibliográfica realizada por Bravo, Uribe y Contreras, encontraron que "las enfermeras brindan escaso tiempo de apoyo a las madres, entendido como la promoción del confort, el apoyo emocional, la entrega de información y la abogacía, reportando que los profesionales estudiados ocuparían, en su jornada laboral, solo el 12,4 \% del 
tiempo" (44); las enfermeras refirieron que esto sucedía debido a la carga laboral y a la ausencia de un cuidado integral; el estudio también indica que las enfermeras pueden actuar de dos formas en el acompañamiento en el trabajo de parto: desde lo físico y desde lo relacional, la última incluye empatía, compasión y palabras suaves que alienten a la madre (44).

\section{DISCUSIÓN}

Los requerimientos de humanización en la atención y salud se están implementando en muchas instituciones siendo el cuidado humanizado prioritario en los planes de mejora de muchos hospitales (45), pero es necesario que el personal de enfermería realice introspección, que reconozca la importancia de su quehacer el cual implica brindar cuidados basados en la empatía y en el conocimiento científico; esto para sustentar que no debería ser necesario que entidades externas $u$ otras profesiones, establezcan lo que debería hacer la enfermería en cuanto al cuidado (46). Una de las grandes falencias que se pueden evidenciar actualmente en el cuidar es la falta de acompañamiento. La enfermería se ha visto afectada por las exigencias de las instituciones en las que se ejerce, en donde su campo de acción se ha desplazado a las actividades administrativas del cuidado y la administración de medicamentos; por lo tanto, el cuidado y el acompañamiento directo ha pasado a manos de los auxiliares de enfermería.
De igual forma, es necesario que las áreas administrativas y la gerencia de los servicios de la salud conozcan y reconozcan el quehacer de enfermería con respecto al cuidado, pues para que haya cuidado humanizado, estas áreas administrativas deben ampliar las oportunidades para que los profesionales de la enfermería se puedan dedicar al cuidado directo y no les impongan otras funciones que desvirtúan su quehacer. Un estudio respalda esta afirmación, pues en él, las enfermeras revelan "que ellas también requieren que los directores reconozcan y valoren sus conocimientos y su experiencia" (47).

Muchas instituciones, al tener una visión muy empresarial con respecto a los servicios de salud, desenfocan el cuidado impidiendo que a las pacientes se les pueda brindar en las salas de parto acompañamiento y cuidado holístico; les asignan a los enfermeros(as) otro tipo de actividades que implican tiempo, disminuyendo las posibilidades de establecer una adecuada relación enfermera(o)-paciente y desconociendo la ardua preparación del profesional en enfermería con respecto al cuidado (48). De esta forma, podría inferirse que las instancias administrativas de muchas instituciones de salud desconocen los pronunciamientos de la OMS cuando dice que "desde 1996, la Organización Mundial de Salud (OMS) consideró al personal profesional de enfermería como un elemento estratégico para lograr un cambio en la calidad y la eficacia de los servicios de atención 
obstétrica" (49). De igual forma, la OMS "desde el año 1999 se instó a los Estados miembros al fortalecimiento y potencialización del desarrollo de servicios de enfermería creando modelos para la atención humanizada y de calidad en la atención del embarazo, parto, puerperio y recién nacido de bajo riesgo"(30), todo esto con el fin de generar un cambio en el paradigma de la atención obstétrica, buscando que la mujer se convierta en el centro del cuidado y atención, orientada a la prevención con acciones sencillas y de bajo costo, brindando seguridad y calidad en la atención(30).

De esta forma, es imprescindible resaltar que la sociedad valora el cuidado de enfermería, y ha reconocido la importancia de los profesionales en esta área para lograr disminuir la mortalidad materna, evidenciándose así en la modificación realizada a la ruta integral materno-perinatal, en la cual se determina que los profesionales en enfermería son el talento humano indicado para la atención del parto de bajo riesgo; el requerimiento mínimo de talento humano para la atención de este tipo de partos es de un profesional en medicina, o profesional en enfermería que certifique formación específica para atención de partos de baja complejidad, en pregrado o en posgrado, expedida por una institución de educación superior reconocida por el Estado, donde se ofrezca el programa de enfermería (50). Es así como el fortalecimiento de la enfermería puede disminuir la morbimortalidad materno-perinatal, siendo necesario que todos los profesionales de enfermería tomen consciencia de su importancia en el cuidar, en el acompañar y en la sociedad en general (51).

\section{CONCLUSIONES}

Mediante la revisión bibliográfica se encuentra el impacto positivo del acompañamiento por parte del profesional en enfermería durante el parto, disminuyendo el uso tanto de inductores como de procedimientos invasivos, igualmente, disminuyendo la morbimortalidad materna y perinatal; además, el acompañamiento del profesional en enfermería aumenta los partos satisfactorios y la tranquilidad de la madre, ofreciendo un parto humanizado y sustentado en el conocimiento científico, holístico y ético, basado en el respeto por la gestante y por sus derechos y dignidad como mujer.

Se encuentra muy necesario el rol de la enfermería durante el trabajo de parto, no solo para la gestante y su hijo por nacer sino también para su familia, brindando apoyo, comprensión y educación para esta etapa. En los diferentes artículos se evidencia que la enfermería es la encargada de velar porque se respeten los derechos de la madre, tanto en el caso del personal de salud como de personas externas que tengan algún tipo de contacto con ella durante el trabajo de parto y parto. De igual forma, mediante el análisis de la biblio- 
grafía se identifica que existen riesgos para las mujeres en gestación cuando hace falta personal capacitado en un trato más humano durante el parto; es así como las entidades gubernamentales en algunos países han intentado implementar programas adecuados para la formación y actualización de enfermeros obstétricos, para evitar la mortalidad en la mujer en trabajo de parto.

Financiamiento

\section{CONFLICTO DE INTERESES}

La autora declara que no existe conflicto de intereses.

\section{FINANCIACIÓN}

Fundación Universitaria del Área Andina.

\section{REFERENCIAS}

1. Vélez Álvarez, C; Vanegas García, JH. El cuidado en enfermería, perspectiva fenomenológica. Revista Hacia la Promoción de la Salud [Internet]. 2011 [consultado el 18 de diciembre de 2018];16(2):175-189. Disponible en: http://www. scielo.org.co/pdf/hpsal/v16n2/v16n2a13.pdf

2. Báez-Hernández, F., Nava-Navarro, V., Ramos-Cedeño, L., \& Medina-López, O. El significado de cuidado en la práctica profesional de enfermería. Aquichan 9(2), [Internet]. 2009 [consultado el 8 noviembre de 2017]. Disponible en: http://aqui- chan.unisabana.edu.co/index.php/aquichan/article/ view/1476/1676

3. Por la cual se reglamenta la profesión de enfermería en Colombia y se dictan otras disposiciones. Ley 266/1996. Pub. Diario Oficial No. 42710 (05-02-1996). [Internet]. 1996 [consultado el 8 de noviembre de 2017]. Disponible en: https://www. mineducacion.gov.co/1621/articles-105002_ archivo_pdf.pdf.

4. Daza-Caballero R; Medina LE. Significado del cuidado de enfermería desde la perspectiva de los profesionales de una institución hospitalaria de tercer nivel en Santafé de Bogotá, Colombia. Cultura de los Cuidados. 19 [Internet].2006 [consultado el 10 noviembre de 2017]. Disponible en: https://rua.ua.es/dspace/bitstream/10045/968/1/ culturacuidados_19_08.pdf.

5. Quintero MT; Gómez M. El cuidado de enfermería significa ayuda. Aquichan [Internet] 2010 [consultado 26 de mayo 2019];10(1). Disponible en: http://www.scielo.org.co/pdf/aqui/v10n1/ v10n1a02.pdf.

6. Leininger $M$, McFarland $M$. Transcultural Nursing: concepts, theories, research, and practice. Third edition. Madeleine Leininger. The Theory of Culture Care and the Ethnonursing. New York: Mcgraw-Hill. 2002. 
7. Durán de Villalobos MM. El cuidado: pilar fundamental de enfermería. Avances de Enfermería [Internet]. 1994 [consultado el 10 de noviembre de 2017];12(1):16-23. Disponible en: https://revistas.unal.edu.co/index.php/avenferm/article/ view/16549/17462.

8. Díaz-Teruel V. Relación lógica entre cuidado y acompañamiento en las etapas de la vida. Revista de Enfermería [Internet]. 2016 [consultado el 13 noviembre 2017];10(2). Disponible en: http:// www.ene-enfermeria.org/ojs/index.php/ENE/ article/view/639/acompa\%C3\%B1 amiento.

9. De León V.A. Humanización del cuidado de enfermería en la atención del parto en el hospital regional de occidente [trabajo de grado]. Quetzaltenango: Universidad Rafael Landívar; 2015 [consultado el 14 de noviembre 2017]. p. 14-21. Disponible en: http://recursosbiblio.url.edu.gt/tesiseortiz/2015/09/02/De-Leon-Antonieta.pdf.

10. De MolinaFernández, I. Rubio-Rico, L., Roca-Biosca, A., Jimenez-Herrera, M., De la Flor-Lopez, M., \& Sirgo, A. Ansiedad y miedos de las gestantes ante el parto: La importancia de su detección. Revista Portuguesa de Enfermagem de Saúde Mental [Internet]. 2015 [consultado el 14 noviembre 2017];(13):17-32. Disponible en: http://www.scielo.mec.pt/scielo.php?script=sci_abstract\&pid=S1647-21602015000200003\&I$\mathrm{ng}=\mathrm{pt} \& \mathrm{nrm}=$ iso $\&$ tlng $=\mathrm{es}$
11. Alemany, M. Velasco J. Aspectos emocionales que rodean el nacimiento: estado de la cuestión. Matronas profesión [Internet]. 2005 [consultado el 15 noviembre 2017];6(4):23-27. Disponible en: https://www.federacion-matronas.org/wp-content/uploads/2018/01/vol6n4pag23-27.pdf.

12. Secretaría Distrital de Salud: Guía de cuidado de enfermería a la familia gestante durante el trabajo de parto y el parto. Enfermería basada en la evidencia (EBE) [Internet]. Imprenta Distrital; [consultado el 20 de enero 2019]. Disponible en: http://www.saludcapital.gov.co/DDS/Guas $\% 20$ de\%20cuidado\%20de\%20enfermera/Guia\%20 Sala\%20de\%20partos.pdf.

13. Terán P, Castellanos C, González-Blanco M, Ramos D. Violencia obstétrica: percepción de las usuarias. Rev Obstet Ginecol Venez [Internet]. 2013 [consultado el 15 noviembre 2017];73(3):171-180. Disponible en: http://www. scielo.org.ve/pdf/og/v73n3/art04.pdf.

14. Tamayo Muño J, Restrepo Moreno C.M., Gil L., González Vélez A.C. Violencia obstétrica y aborto. Aportes para el debate en Colombia. Grupo Médico por el Derecho a Decidir - Colombia Red Global Doctors for Choice [Internet]. 2015 [consultado el 13 de mayo de 2017]. Disponible en: https://www.safeabortionwomensright.org/ wp-content/uploads/2016/02/GDC_Obstetric-Violence_ES-1.pdf 
15. Por la cual se dictan normas de sensibilización, prevención y sanción de formas de violencia y discriminación contra las mujeres, se reforman los Códigos Penal, de Procedimiento Penal, la Ley 294 de 1996 y se dictan otras disposiciones. Ley 1257/ 2008. Pub. Diario Oficial 47193 (04-12-2008). Disponible en: http://www.sdp.gov.co/sites/default/ files/anexo_5_ley_1257_del_2008.pdf.

16. Camacaro, M, Ramírez, M, Lanza, L, Herrera, $M$. Conductas de rutina en la atención al parto constitutivas de violencia obstétrica. Utopía y Praxis Latinoamericana [Internet]. 2015 [consultado 3 de mayo 2017];20(68):113-120. Disponible en: https://www.redalyc.org/articulo. oa?id=27937090009.

17. Veliz Rojas, L.- Bianchetti Saavedra, A. Acompañamiento y cuidado holístico de enfermería en personas con enfermedades crónicas no adherentes al tratamiento. Revista electrónica Enfermería actual en Costa Rica [Internet]. 2017 [consultado 28 de abril 2017];32:1-11. Disponible en: http://www.scielo.sa.cr/pdf/enfermeria/ n32/1409-4568-enfermeria-32-00186.pdf.

18. Ministerio de Salud - Dirección General de Promoción y Prevención. Norma técnica para la atención del parto [Internet]. 2000. Disponible en: https://www.minsalud.gov.co/sites/rid/ Lists/BibliotecaDigital/RIDE/VS/PP/3Atencion\%20 del\%20Parto.pdf.
19. Fernández Raigada RI, Satisfacción materna con el parto y puerperio relacionada con la atención hospitalaria [trabajo de grado]. Oviedo: Universidad de Oviedo; 2013 [consultado el 27 de marzo de 2017]. Disponible en: http://digibuo. uniovi.es/dspace/bitstream/10651/28566/6/ TFG\%20RosaFernandezRaigada.pdf.

20. Molina Restrepo, ME. El modelo de salud colombiano y la calidad del cuidado de enfermería. Aquichan [Internet]. 2006 [consultado el 27 de marzo de 2017]; 6(1):148-155. Disponible en: https://www.redalyc.org/articulo. oa?id=74160115.

21. Valencia López, V. Revisión documental en el proceso de investigación [Internet]. Pereira: Universidad Tecnológica de Pereira. Disponible en: https://univirtual.utp.edu.co/pandora/recursos/1000/1771/1771.pdf.

22. Por la cual se establecen las normas científicas, técnicas y administrativas para la investigación en salud. Resolución número 8430/1993. (04-10-1993). Disponible en: https://www.minsalud.gov.co/sites/rid/Lists/BibliotecaDigital/RIDE/ DE/DIJ/RESOLUCION-8430-DE-1993.PDF.

23. Lagos Jiménez G. Correlación del acompañamiento en las parturientas durante el período expulsivo en el centro materno infantil César López Silva- Villa el Salvador. [trabajo de grado]. Lima: Universidad de San Martín de Porres; 2015 [con- 
sultado el 03 de abril de 2017]. Disponible en: http://www.repositorioacademico.usmp.edu.pe/ bitstream/usmp/1566/3/lagos_jg.pdf.

24. De Oliveira-Sanfelice CF, De Souza-Freitas AF, Separavich-Pregnolatto O, Gonçalves da Silva M, Keiko-Kakuda A. Do parto institucionalizado ao parto domiciliar. Relato de Experiência. Rev Rene [Internet]. 2014 [consultado el 03 de mayo de 2017];15(2):362-70. Disponible en: http://periodicos.ufc.br/rene/article/view/3170.

25. Saavedra Infante, DL. Comportamientos de cuidado del profesional de enfermería que percibe la gestante en trabajo de parto y parto en la clínica Palermo [tesis de maestría]. Colombia: Universidad Nacional de Colombia; 2013 [consultado el 11 de abril de 2017] Disponible en: http:// www.bdigital.unal.edu.co/11168/1/deisylorenasaavedrainfante.2013.pdf.

26. Quevedo P. Violencia obstétrica: una manifestación oculta de la desigualdad de género [trabajo de grado]. Mendoza, Argentina: Universidad Nacional de Cuyo [Internet]. 2012 [consultado el 16 de abril de 2017]. Disponible en: http://bdigital.uncu.edu. ar/objetos_digitales/4785/tesisquevedo.pdf.

27. Mahlmester LR. Best practices in perinatal nursing: "speaking up"--a positive approach to improving outcomes when obstetrical emergencies arise. J Perinat Neonatal Nurs 21 [Internet]. 2007 [consultado el 19 de marzo de 2017];(4):282-4.
Disponible en: https://insights.ovid.com/pubme$\mathrm{d}$ ?pmid $=17700092$.

28. Pérez ME. La enfermera como educadora de la embarazada y su núcleo familiar. Rev. Hosp. Mat. Inf. Ramón Sardá [Internet]. 1996 [consultado el 29 de marzo de 2017];15(3). Disponible en: http://www.sarda.org.ar/content/download/471/2544/file/102-106.pdf.

29. Giraldo Montoya Dora Isabel, González Mazuelo Elvira Margarita, Henao López Claudia Patricia. Experiencias de las mujeres durante el trabajo de parto y parto. av.enferm. [Internet]. 2015 [consultado el 17 de abril de 2017];33(2):271-281. doi: 10.15446/av.enferm. v33n2.4227.

30. Ramírez-Hernández C. Modelo de atención obstétrica por enfermeras obstetras y perinatales en el Hospital General de Cuautitlán: una experiencia exitosa. Rev CONAMED [Internet]. 2012 [consultado el 19 de mayo de 2017]; 17 (1):3741. Disponible en: https://dialnet.unirioja.es/ servlet/articulo? codigo $=4114330$.

31. Reis CS. et al. Análise de partos acompanhados por enfermeiras obstétricas na perspectiva da humanização do parto e nascimento Analysis of births attended by nurse midwives under the perspective of humanization of childbirth. Revista de Pesquisa: Cuidado é Fundamental 
[Internet]. 2016 [consultado el 18 de mayo de 2017];8(4):4972-9. Disponible en: http://www. seer.unirio.br/index.php/cuidadofundamental/ article/view/3966/pdf.

32. Oria Vicharra C.M. Influencia del apoyo del acompañante en el proceso del trabajo de parto en el Instituto Nacional Materno Perinatal [trabajo de grado]. Lima: Universidad Nacional Mayor de San Marcos; 2007 [consultado el 22 de marzo de 2017]. Disponible en: http://cybertesis.unmsm. edu.pe/bitstream/handle/cybertesis/1149/Oria_ vc. pdf? sequence $=1$ \&isAllowed $=\mathrm{y}$.

33. Gómes de Oliveira JD; Correia-Campos T Neri; De Lima Costa Souza FM, Barbosa Dayim RM, De Costa Dantas J. Obstetric nurses' perception in assistance to the parturient. Journal of Nursing. [Internet]. 2016 [consultado el 13 de marzo de 2017]; 10(10):3868-75. doi: 10.5205/1981-8963-v10i10 a11454p3868-3875-2016..

34. González-Burgos JT; Quintero-Martínez DC. Percepción del cuidado humanizado en pacientes que ingresan al servicio de hospitalización de urgencias de Ginecobstetricia de una Institución de II y III Nivel de atención [trabajo de grado]. Bogotá: Universidad Javeriana; 2009 [consultado el 22 de mayo de 2017]. Disponible en: https://repository. javeriana.edu.co/bitstream/handle/10554/9730/ tesis05-3. pdf;jsessionid =78C481F6D1098D4E9FC7221 C9C152ACE? sequence $=1$.
35. Morláns Lanau M. El acompañamiento continúo en los partos instrumentales: resultados obstétricos y perinatales, nivel de satisfacción de la mujer y acompañante. Reduca (Enfermería, fisioterapia y podología). Serie Matronas. [Internet]. 2011[consultado el 29 de marzo de 2017];3(3):176-206. Disponible en: http://revistareduca.es/index.php/reduca-enfermeria/article/ view/740/755.

36. Rámila-Gómez M, Gil Vázquez L. Rodríguez-Santana M. Importancia del acompañamiento continuo en cesáreas programadas: ansiedad materna. Biblioteca Las Casas [Internet]. 2016 [consultado el 16 de abril de 2017];12(3). Disponible en: http://www.index-f.com/lascasas/ documentos/lc0919.php.

37. Vargens O, Silva A., Progianti JM. Contribuição de enfermeiras obstétricas para consolidação do parto humanizado em maternidades no Rio de Janeiro-Brasil. Escola Anna Nery [Internet]. 2017 [consultado el 17 de abril de 2017]; 21(1). doi: 10.5935/1414-8145.20170015.

38. Contreras M; Guaymás M. Cuidados de enfermería para un trabajo de parto humanizado. Notas de enfermería [Internet]. 2017 [consultado el 15 de mayo de 2017];29:11-12. Disponible en: http://www.sanatorioallende.com/FILES/Archivos/docs/2018/Revista\%20n\%C2\%B029/4\%20 Cuidados\%20de\%20enfermer\%C3\%ADa\%20 
para\%20un\%20trabajo\%20de\%20parto\%20humanizado.pdf.

39. Mattos de Souza R, Santos-Soares L, Baptista-Quitete J. Home parturition: power to feminine nature and a challenge for the obstetric nurse. Revista Online de Pesquisa: Cuidado é Fundamental [Internet]. 2014 [consultado el 16 de mayo de 2017];6(1):118-131. Disponible en: http://www. seer.unirio.br/index.php/cuidadofundamental/ article/view/2260/pdf_1099.

40. López-Villar C, Soraluce-Acebo A. Parto activo y acompañamiento respetuoso. En: López-Villar C, coordinador. Estudios multidisciplinares para la humanización del parto. Jornadas Universitarias Multidisciplinares para la Humanización del Parto. España: Universidad de Coruña; [Internet]. 2011 [consultado el 16 de mayo de 2017]; p. 147162. Disponible en: https://ruc.udc.es/dspace/ handle/2183/9086? locale-attribute $=$ es.

41. Patrocinio-Sandoval RDP. Nivel de satisfacción de la puérpera sobre el cuidado integral que brinda la enfermera en el servicio de ginecoobstetricia del Hospital Nacional Arzobispo Loayza [tesis]. Perú: Universidad nacional mayor de San marcos; 2013 [consultado el 14 de marzo de 2017]. Disponible en: http://cybertesis.unmsm. edu.pe/bitstream/handle/cybertesis/3985/Patrocinio_sr.pdf? sequence $=1$ \&isAllowed $=y$.
42. Vela-Coral GDP. Percepción de la paciente sobre la atención del parto humanizado en el Servicio de Centro Obstétrico del Instituto Nacional Materno Perinatal durante el periodo marzo-abril de 2015 [tesis]. Perú: Universidad nacional mayor de San marcos. 2015 [consultado el 12 de enero de 2019]. Disponible en: http://cybertesis.unmsm. edu.pe/bitstream/handle/cybertesis/4278/Vela_ cg. pdf? sequence $=1$.

43. Coral Ibarra R. Humanización del cuidado de enfermería durante el parto. Revista U.D.C.A. [Internet]. 2008 [consultado el 15 de marzo de 2019];11(1):47-56. Disponible en: https://revistas.udca.edu.co/index.php/ruadc/article/view/601

44. Bravo V Paulina, Uribe T Claudia, Contreras M Aixa. El cuidado percibido durante el proceso de parto: una mirada desde las madres. Rev. chil. obstet. ginecol. [Internet]. 2008 [consultado el 21 de abril de 2017];73(3):179-184. doi: 10.4067/ s0717-75262008000300007.

45. Ministerio de Salud y Protección Social [Internet]. Bogotá: Imprenta Nacional de Colombia; 2016. Plan Nacional de Mejoramiento de la Calidad en Salud, 2016-2021 [consultado el 21 de febrero de 2018]. Disponible en: https://www.minsalud. gov.co/sites/rid/Lists/BibliotecaDigital/RIDE/DE/CA/ Plan-nacional-de-mejoramiento-calidad.pdf. 
46. Poblete-Troncoso M, Valenzuela- Suazo S. Cuidado humanizado: un desafío para las enfermeras en los servicios hospitalarios. Acta Paul Enferm [Internet]. 2007 [consultado el 24 de abril de 2017];20(4):499-503. Disponible en: http:// www.scielo.br/pdf/ape/v20n4/18.pdf.

47. Tapp-Dianne SK, Stewart J. La autonomía en la práctica de enfermería. Aquichán [Internet]. 2005 [consultado el 16 de marzo de 2017];5(1):114127. Disponible en: http://aquichan.unisabana. edu.co/index.php/aquichan/article/view/65/135.

48. González-Castro LF, Moreno-Martínez IA, García-Mancipe M, Vélez-Ferreira $M$. La relación entre profesionales de enfermería y medicina: una posible explicación de los resultados. Med. UNAB [Internet]. 2010 [consultado el 24 de febrero de 2018];13(1). Disponible en: http://132.248.9.34/ hevila/Medunab/2010/vol13/no1/3.pdf.

49. Oviedo-Zúñiga AM, Rodríguez-García C, Ruíz-Martínez AO, De Lourdes-Vargas Santillán M. Cuidados del control prenatal en el primer nivel de atención en relación a la NOM-007-SSA2-1993 [Inernet]. Universidad Autónoma del Estado de México.RI. 2016 [consultado el 22 de febrero de 2018]. Disponible en: http://ri.uaemex.mx/bitstream/handle/20.500.11799/40660/AMOZ\%20 ART\%C3\%8DCULO\%20DEFINITIVO\%20WAXAPA. pdf? sequence $=1$.
50. Consultor Salud [Internet]. Ruta de atención en salud materno perinatal [actualizado 2018 mayo 14]; [consultado el 24 de febrero de 2018]. Disponible en: http://www.consultorsalud.com/ rutas-integrales-de-promocion-de-la-salud-y-materno-perinatal-resolucion-y-lineamientos.

51. Hernández-Betancur Ana María, Vásquez-Truisi Martha Lucía. El cuidado de enfermería comprometido: motor en la satisfacción de la gestante durante el control prenatal. Univ. Salud [Internet]. 2015 June [consultado el 26 de febrero de 2018];17(1):80-96. Disponible en: http://www. scielo.org.co/scielo.php?script $=$ sci_arttext\&pi$d=$ S0124-71072015000100008\&lng=en

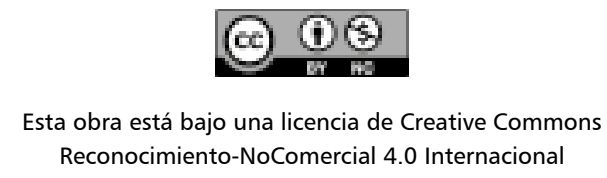

sta obra está bajo una licencia de Creative Commons 